\title{
Perfil antropométrico e variáveis associadas de pacientes internados em uma unidade
}

\section{de terapia intensiva pediátrica}

\author{
Anthropometric profile and associated variables of patients admitted to a pediatric intensive care \\ unit
}

Perfil antropométrico y variables asociadas de los pacientes ingresados en una unidad de cuidados

intensivos pediátricos

Recebido: 22/09/2021 | Revisado: 27/09/2021 | Aceito: 19/10/2021 | Publicado: 21/10/2021

\author{
Laís Santos Calmon \\ ORCID: https://orcid.org/0000-0002-9660-2289 \\ Universidade do Estado da Bahia, Brasil \\ E-mail: laiscalmon@hotmail.com \\ Adriele Conceição da Silva \\ ORCID: https://orcid.org/0000-0003-2613-3672 \\ Hospital Geral Roberto Santos, Brasil \\ E-mail: adrielec.silva@yahoo.com.br \\ Luana de Oliveira Leite \\ ORCID: https://orcid.org/0000-0002-3031-8576 \\ Universidade do Estado da Bahia, Brasil \\ E-mail: loleite@uneb.br \\ Lílian Brito da Silva Fatal \\ ORCID: https://orcid.org/0000-0001-7613-4775 \\ Universidade do Estado da Bahia, Brasil \\ E-mail: 1fatal@uneb.br
}

\begin{abstract}
Resumo
Introdução: O diagnóstico da desnutrição e dos sinais de riscos nutricionais, se identificados, são fundamentais para a intervenção dietoterápica individualizada em pacientes criticamente doentes, a fim de garantir uma melhora no estado nutricional e desfechos clínicos desses pacientes. Objetivo: avaliar o estado antropométrico dos pacientes internados em uma UTIP, bem como verificar a sua associação com a idade, tempo de internamento e desfechos clínicos. Métodos: Estudo observacional, transversal e retrospectivo. A amostra foi composta por 88 pacientes internados no período de fevereiro a julho de 2019. Foram realizadas as avaliações do estado antropométrico dos pacientes. As variáveis coletadas incluíram dados demográficos, diagnóstico clínico, tempo de internamento e motivo da alta do paciente. As análises foram realizadas no programa SPSS versão 20.0. As variáveis categóricas foram apresentadas em frequências absolutas e relativas, e as contínuas, em medianas e intervalo interquartil. Para avaliar a associação entre as variáveis, foi realizado o teste qui-quadrado. Resultados: Segundo a avaliação antropométrica dos pacientes $63,2 \%$ eram eutróficos, $8,1 \%$ apresentavam excesso de peso e $28,7 \%$ eram desnutridos, destes $68 \%$ eram desnutridos graves. Não houve associação estatisticamente significante entre as variáveis analisadas. Conclusão: A avaliação do estado antropométrico dos pacientes críticos internados na UTI Pediátrica no presente estudo demonstrou elevado percentual de crianças desnutridas e não houve associação estatisticamente significante entre o estado antropométrico, idade, tempo de internamento e os desfechos tempo de internamento e óbito.
\end{abstract}

Palavras-chave: Estado antropométrico; Avaliação nutricional; Unidade de terapia intensiva pediátrica.

\begin{abstract}
Introduction: The diagnosis of malnutrition and signs of nutritional risk, if identified, are fundamental for individualized dietary intervention in critically ill patients to ensure an improvement in nutritional status and clinical outcomes of these patients. Objective: To evaluate the anthropometric status of patients admitted to a PICU, as well as to verify its association with age, length of stay and clinical outcomes. Methods: Observational, cross-sectional and retrospective study. The sample was composed of 88 patients hospitalized from February to July 2019. Assessments of the anthropometric status of the patients were performed. Variables collected included demographic data, clinical diagnosis, length of stay, and reason for patient discharge. Analyses were performed in the SPSS version 20.0 program. Categorical variables were presented as absolute and relative frequencies, and continuous variables as medians and interquartile ranges. To evaluate the association between variables, the chi-square test was used. Results: According to the anthropometric evaluation of the patients $63.2 \%$ were eutrophic, $8.1 \%$ were overweight and $28.7 \%$ were malnourished, of these $68 \%$ were severely malnourished. There was no statistically significant association
\end{abstract}


between the variables analyzed. Conclusion: Evaluation of the anthropometric status of critically ill patients admitted to the Pediatric ICU in this study showed a high percentage of malnourished children and no statistically significant association between anthropometric status, age, length of stay and the outcomes length of stay and death.

Keywords: Anthropometric status; Nutritional assessment; Pediatric intensive care unit.

\section{Resumen}

Introducción: El diagnóstico de la desnutrición y las señales de riesgo nutricional, si se identifican, son fundamentales para la intervención dietética individualizada en pacientes críticamente enfermos, con el fin de garantizar una mejora del estado nutricional y de los defectos clínicos de estos pacientes. Objetivo: Evaluar el estado antropométrico de los pacientes ingresados en una UCIP, así como verificar su asociación con la edad, la duración de la estancia y los resultados clínicos. Métodos: Estudio observacional, transversal y retrospectivo. La muestra estuvo compuesta por 88 pacientes hospitalizados en el periodo de febrero a julio de 2019. Se realizaron evaluaciones del estado antropométrico de los pacientes. Las variables recogidas fueron los datos demográficos, el diagnóstico clínico, la duración de la estancia y el motivo del alta del paciente. Los análisis se realizaron en el programa SPSS versión 20.0. Las variables categóricas se presentaron como frecuencias absolutas y relativas y las continuas como medianas y rango intercuartil. Para evaluar la asociación entre variables, se realizó la prueba de chi-cuadrado. Resultados: Según la evaluación antropométrica de los pacientes, el 63,2\% eran eutróficos, el 8,1\% tenían sobrepeso y el 28,7\% estaban desnutridos, de los cuales el $68 \%$ estaban gravemente desnutridos. No hubo ninguna asociación estadísticamente significativa entre las variables analizadas. Conclusión: La evaluación del estado antropométrico de los pacientes críticos ingresados en la UCI pediátrica en este estudio mostró un alto porcentaje de niños desnutridos y no hubo una asociación estadísticamente significativa entre el estado antropométrico, la edad, la duración de la estancia y los resultados duración de la estancia y muerte.

Palabras clave: Estado antropométrico; Evaluación nutricional; Unidad de cuidados intensivos pediátricos.

\section{Introduçãa}

A avaliação do estado nutricional em pediatria é um dos principais fatores para identificação do estado de saúde na infância, possibilitando investigar se uma criança está se desenvolvendo dentro dos padrões recomendados ou está se afastando dos mesmos, devido às doenças ou às condições desfavoráveis de sobrevida (Ferreira, 2000).

A desnutrição infantil é decorrente do desequilíbrio entre a necessidade energética e a ingestão inadequada de nutrientes, o que gera déficits acumulados de energia, proteína ou micronutrientes, prejudicando o adequado crescimento e desenvolvimento (Bechard et al., 2016). O diagnóstico da desnutrição e dos sinais de riscos nutricionais, se identificados, são fundamentais para a intervenção dietoterápica individualizada em pacientes criticamente doentes (Silva \& Tiengo, 2014).

Atualmente, a desnutrição é um desafio para o sistema de saúde, afetando pelo menos uma em cada três pessoas no mundo. Segundo dados da Organização Mundial de Saúde (OMS) de 2018, a desnutrição colabora para 3,1 milhões (45\% do total) de mortes em crianças menores de 5 anos todos os anos e 151 milhões $(22,2 \%)$ de crianças menores de 5 anos de idade apresentam baixa estatura para idade (WHO, 2018). A desnutrição infantil ainda possui alta prevalência nos hospitais, apesar da redução a nível mundial (Rocha et al., 2006, Ribeiro et al., 2018).

As crianças hospitalizadas geralmente apresentam taxa metabólica basal elevada e as reservas de energia são geralmente limitadas nestas condições, aumentando o risco de desenvolver desnutrição durante o curso de uma doença (Silva et al., 2021). Estudos realizados em âmbito hospitalar ainda apontam para alta prevalência de desnutrição na admissão (Sarni et al, 2009, Pawellek et al, 2008). Está é considerada um fator de risco para maior morbimortalidade, atraso de recuperação, complicações respiratórias, pós cirúrgicas e infecciosas, gerando maiores custos relacionados aos cuidados em saúde e aumento no tempo de permanência hospitalar quando comparados aos pacientes com estado nutricional adequado (Silva et al., 2021, Mesquita et al, 2008, Bejon et al, 2008).

Em ambiente hospitalar, a avaliação antropométrica é um método eficaz e não oneroso, podendo ser útil na maioria dos casos. O correto diagnóstico nutricional, obtido através da avaliação adequada apoia o diagnóstico clínico, facilita o prognóstico, permitindo uma intervenção precoce e segura (Sarni et al., 2009). Assim, destaca-se como fator importante para ser realizado em unidade de terapia intensiva pediátrica (UTIP), a fim de se garantir uma adequação da terapia nutricional e 
melhora no estado nutricional e possíveis agravos devido ao comprometimento nutricional (Silva \& Tiengo, 2014).

O objetivo deste estudo foi avaliar o estado antropométrico dos pacientes internados em uma UTIP, bem como verificar a sua associação com a idade, tempo de internamento e desfechos clínicos.

\section{Metodologia}

Trata-se de um estudo observacional, transversal e retrospectivo (Pereira et al. 2018), com pacientes de uma a UTIP de um hospital público de Salvador -BA, internados no período de fevereiro a julho de 2019. A coleta de dados ocorreu de outubro a dezembro de 2019. As informações foram coletadas a partir dos prontuários clínicos (evolução médica e evolução da nutrição), e de mapas de prescrição dietoterápica, pertencentes ao Serviço de Nutrição e Dietética do hospital. Foram incluídos na pesquisa os pacientes com idade igual ou maior que 29 dias até 18 anos e excluídos os pacientes com ascite, edema, amputados, e que não tenham sido encontrados os registros da estatura e/ou peso. Em caso de mais de um internamento na unidade no período de coleta de dados, foi utilizado como base o primeiro internamento deste paciente e foram desconsideradas as demais internações.

Dos pacientes que atenderam os critérios de inclusão coletaram-se os seguintes dados: nome, data de nascimento, data da admissão, data da alta, sexo (feminino ou masculino), idade (meses), peso (gramas), altura (centímetros), diagnóstico clínico, tipo de internação e motivo da alta do paciente.

$\mathrm{Na}$ avaliação dos dados antropométricos, usou-se software WHO Anthro 3.1.0 (para 0 a 5 anos) e WHO AnthroPlus 1.0.2 (para maiores de 5 a 19 anos). O estado antropométrico foi avaliado através do escore z dos índices peso/idade, estatura/idade, peso/estatura e Índice de massa corporal (IMC) /idade e a sua classificação a partir dos critérios da Organização Mundial da Saúde (OMS), 2006 para menos de 5 anos e OMS, 2007 para pacientes com 5 anos ou mais. As crianças com idade gestacional menor que 37 semanas tiveram sua idade atual corrigida até dois anos. Para os pacientes com diagnóstico de desordens neurológicas foram utilizadas as curvas de paralisia cerebral propostas por Brooks et al, 2011. A avaliação do estado antropométrico a partir das curvas foi realizada através dos percentis. Para as análises estatísticas, as variáveis desnutrição energético proteica (DEP) e DEP grave foram agrupadas em DEP e as variáveis sobrepeso e obesidade em excesso de peso.

Para análise estatística foi utilizado o software Statistical Packadge Social of Sciences (SPSS) para Windows (versão 20.0). As variáveis categóricas foram apresentadas em frequências absolutas e relativas, e as contínuas, em medianas e intervalo interquartil, após teste de normalidade de Kolmogorov-Smirnov. Em seguida, para avaliar a associação entre estado antropométrico, prematuridade e motivo da alta, foi realizado o teste qui-quadrado e para avaliar a igualdade das medianas da idade e tempo de internamento entre as categorias de estado antropométrico, foi utilizado o teste de Kruskal Wallis. O nível de significância considerando para correlações foi $p$-valor $\leq 0,05$.

O projeto foi aprovado pelo Comitê de Ética em Pesquisa da Universidade do estado da Bahia (n. ${ }^{\circ}$ 3.561.969), em setembro de 2019, de acordo com a Resolução 466/12 do Conselho Nacional de Saúde.

\section{Resultados}

Dos 87 pacientes estudados, 60,9\% (53) eram do sexo masculino, 17,2\% (15) prematuros, com mediana de idade de 16 meses. O motivo de internação da maioria dos pacientes foi clínico (77\%), e as disfunções orgânicas mais prevalentes foram neurológica $(33,3 \%)$, respiratória $(22,2 \%)$ e cardíaca $(10,1 \%)$. O percentual de óbitos foi de 5,7\% (5). As características dos pacientes internados podem ser observadas nos resultados mostrados na Tabela 1. 
Tabela 1 - Características demográficas e clínicas dos pacientes internados em Unidade de Terapia Intensiva Pediátrica em um hospital público de Salvador, 2021.

\begin{tabular}{|c|c|c|c|}
\hline Variáveis & Categoria & $\mathbf{N}$ & $\%$ \\
\hline \multirow{2}{*}{ Sexo } & Feminino & 34 & 39,1 \\
\hline & Masculino & 53 & 60,9 \\
\hline \multirow{2}{*}{ Prematuro } & Sim & 15 & 17,2 \\
\hline & Não & 72 & 82,8 \\
\hline \multirow{6}{*}{ Diagnóstico clínico } & Respiratória & 22 & 22,2 \\
\hline & Neurológica & 33 & 33,3 \\
\hline & Cardiológica & 10 & 10,1 \\
\hline & Hepática & 6 & 6,1 \\
\hline & Renal & 11 & 11,1 \\
\hline & Outros & 17 & 17,1 \\
\hline \multirow{2}{*}{ Motivo de internamento } & Clínico & 67 & 77 \\
\hline & Cirúrgico & 20 & 23 \\
\hline \multirow{2}{*}{ Motivo da alta } & Melhora clínica & 82 & 94,3 \\
\hline & Óbito & 5 & 5,7 \\
\hline
\end{tabular}

Fonte: Autores.

Segundo a avaliação antropométrica dos pacientes 63,2\% eram eutróficos, $8,1 \%$ apresentavam excesso de peso e $28,7 \%$ eram desnutridos, destes $68 \%$ eram desnutridos graves (Tabela 2 ).

Tabela 2 - Avaliação antropométrica dos pacientes internados em Unidade de Terapia Intensiva Pediátrica em um hospital público de Salvador, 2021.

\begin{tabular}{cccc}
\hline Variável & Categoria & $\mathbf{N}$ & $\%$ \\
\hline \multirow{3}{*}{ Diagnóstico nutricional } & Eutrofia & 55 & 63,2 \\
& Desnutrição & 8 & 9,2 \\
& Desnutrição grave & 17 & 19,5 \\
& Sobrepeso & 5 & 5,8 \\
& Obesidade & 2 & 2,3 \\
\hline
\end{tabular}

Fonte: Autores.

A Tabela 3 mostra que não houve associação entre a idade, tempo de internamento e estado antropométrico dos pacientes. A mediana da idade dos pacientes com baixo peso foi de 14 meses, eutróficos 15 meses e excesso de peso 55 meses. ( p <0,352). Em relação ao tempo de internamento e diagnóstico nutricional foi possível verificar que os pacientes que estavam com baixo peso ficaram internados durante 4,5-14,0 dias e os eutróficos 4,0-15,0 dias ( $p<0,320)$. 
Tabela 3 - Associação entre a idade, tempo de internamento e estado antropométrico dos pacientes internados em uma unidade de terapia intensiva pediátrica de um hospital público em Salvador, 2021.

\begin{tabular}{|c|c|c|c|c|c|}
\hline \multirow[t]{2}{*}{ VARIÁVEL } & \multirow{2}{*}{$\begin{array}{l}\text { GERAL } \\
\text { Mediana } \\
(\mathbf{Q} 1-Q 3)\end{array}$} & \multicolumn{3}{|c|}{ ESTADO ANTROPOMÉTRICO } & \multirow[b]{2}{*}{ Valor de $\mathrm{p}^{x}$} \\
\hline & & $\begin{array}{c}\text { EUTROFIA } \\
\text { Mediana (Q1-Q3) }\end{array}$ & $\begin{array}{c}\text { EXCESSO DE PESO } \\
\text { Mediana (Q1-Q3) }\end{array}$ & $\begin{array}{c}\text { BAIXO PESO } \\
\text { Mediana (Q1-Q3) }\end{array}$ & \\
\hline Idade ** & $16,0(2,0-62,0)$ & $15,0(2,0-57)$ & $55,0(4,0-154)$ & $14(2,0-60,5)$ & 0,352 \\
\hline $\begin{array}{l}\text { Tempo de } \\
\text { internamento } * * *\end{array}$ & $7,0(4,0-14,0)$ & $8,0(4,0-15,0)$ & $6,0(2,0-7,0)$ & $6(4,5-14,0)$ & 0,320 \\
\hline
\end{tabular}

*Test Kruskal Wallis $\quad * *$ Mediana expressa em meses $\quad * * *$ Mediana expressa em dia. Fonte: Autores.

Neste estudo não se constatou associação entre prematuridade, o motivo da alta e estado antropométrico (Tabela 4). Dos pacientes que foram classificados como prematuros, 20,0\% eram desnutridos ou desnutridos graves. Apenas 5 pacientes foram a óbito, destes 40,0\% encontravam-se desnutridos ou desnutridos graves e 60,0\% com eutrofia. Nenhum paciente que estava com excesso de peso foi a óbito. No entanto, não existiu uma associação estatisticamente significante entre o estado nutricional e ser prematuro ( $\mathrm{p}<0,664)$ e ter ido a óbito no internamento $(\mathrm{p}<0,715)$.

Tabela 4 - Associação entre o motivo da alta, prematuridade e estado antropométrico dos pacientes internados em uma unidade de terapia intensiva pediátrica de um hospital público em Salvador, 2021.

\begin{tabular}{|c|c|c|c|c|c|}
\hline \multirow{2}{*}{\multicolumn{2}{|c|}{ VARIÁVEIS }} & \multicolumn{3}{|c|}{ ESTADO ANTROPOMÉTRICO } & \multirow[b]{2}{*}{ Valor de $\mathrm{p}^{*}$} \\
\hline & & \multirow{2}{*}{$\begin{array}{c}\text { EUTROFIA } \\
\mathrm{N}(\%) \\
11(73,3)\end{array}$} & $\begin{array}{c}\text { EXCESSO DE PESO } \\
\mathrm{N}(\%)\end{array}$ & $\begin{array}{c}\text { BAIXO PESO } \\
\mathrm{N}(\%)\end{array}$ & \\
\hline \multirow{2}{*}{ PREMATURO } & SIM & & $1(6,7)$ & $3(20,0)$ & \multirow{2}{*}{0,664} \\
\hline & NÃO & $44(61,1)$ & $6(8,3)$ & $22(30,6)$ & \\
\hline \multirow{2}{*}{$\begin{array}{c}\text { MOTIVO DA } \\
\text { ALTA }\end{array}$} & MELHORA CLÍNICA & $52(63,4)$ & $7(8,5)$ & $23(28,0)$ & \multirow{2}{*}{0,715} \\
\hline & ÓBITO & $3(60,0)$ & $0(0,0)$ & $2(40,0)$ & \\
\hline
\end{tabular}

*Test Qui-Quadrado. Fonte: Autores.

\section{Discussão}

Na população estudada houve maior prevalência de pacientes do gênero masculino. Resultados semelhantes foram encontrados no estudo de Oliveira et al. (2017) no qual o percentual médio foi de $73 \%$ de crianças do sexo masculino. O motivo de internação, na UTIP, da maioria dos pacientes foi clínico com disfunções orgânicas neurológicas. Esse achado pode ser justificado devido ao hospital, onde foi realizado a coleta, ser referência no tratamento de doenças neurológicas.

As medidas de avaliação nutricional antropométrica, peso e estatura, são importantes indicadores de saúde da crianças e as mais utilizadas na avaliação do crescimento, as quais são analisadas de acordo com o sexo e idade da criança (Sigulem et al., 2000, Ista \& Joosten, 2005). Entretanto, para determinar e monitorar o estado nutricional em paciente gravemente doente, ainda não há um "padrão ouro" (Hulst et al., 2004). Nas pesquisas de avaliação do estado nutricional, esses indicadores têm sido amplamente utilizados para diagnóstico nutricional.

O estado nutricional é determinante na recuperação de pacientes pediátricos críticos. A desnutrição neste público é considerada um fator de risco para maior morbimortalidade, atraso de recuperação, complicações respiratórias, pós-cirúrgicas e infecciosas. Estratégias para o diagnóstico nutricional e tratamento precoce da desnutrição em crianças internadas em estado crítico, faz-se necessário, pois permite uma maior assertividade no diagnóstico e terapia nutricional, individualizando o tratamento e obtendo-se evolução desejada (Mesquita et al., 2008, Bejon et al, 2008). 
Neste estudo evidenciou-se que a maioria dos pacientes internados na UTIP estavam eutróficos, no entanto, o percentual observado de pacientes com desnutrição $(28,9 \%)$ ainda é preocupante. A alta prevalência de desnutrição neste público pode estar associada a maior necessidade energética em relação aos adultos, ao estresse metabólico gerado pelo estado patológico e as limitadas reservas de energias (Cameron et al., 2008). Muitos estudos têm demonstrado uma elevada prevalência de desnutrição infantil em pacientes graves (Bechard, 2016, Souza et al., 2012, Jacquot, 2019).

Bechard et al. (2016) encontrou em seu estudo, realizado em crianças internadas na UTIP em uso de ventilação mecânica, um percentual de $46 \%$ dos pacientes com baixo peso. Resultado semelhante foi encontrado em estudo realizado em São Paulo que revelou que 45,5\% das crianças estavam desnutridas na admissão (Souza et al., 2012). Estudos realizados em outros países como França e Alemanha, encontraram um percentual de crianças desnutridas (18,5\% e 21,1\%, respectivamente), mais próximo ao encontrado neste trabalho (Jacquot, 2019, Pawellek et al., 2008).

No estudo de Batista et al. (2021) o tempo médio de permanência na UTIP foi de 11,5 dias (DP $\pm 13,0$ ), em outro estudo realizado em uma UTIP em Fortaleza o tempo de permanência média foi de 15,52 dias (DP \pm 0,94) (Oliveira et al, 2017), valores superiores ao encontrado nesse estudo. Já nos estudos também realizados no Brasil, foi observado tempo médio de internamento de 6,9 dias (Sami et al, 2009) e 8 dias (Silveira et al, 2008) e nos internacionais de Jiménez et al. (2011) (7 dias), Hecht et al. (2014) (4 dias) e Lim et al. (2012) (6,9 dias) mais próximos a mediana de tempo de internamento encontrado nesse estudo (7 dias).

O percentual de pacientes que foram a óbito no internamento na UTIP deste estudo foi baixo (5,7\%), quando comparado a outro estudo realizado no Nordeste que encontrou em 19\% da amostra o desfecho de óbito. No estudo de Batista et al. (2021), 24\% dos pacientes pediátricos evoluíram para óbito. Netto et al. (2014) constataram que a taxa de mortalidade dos pacientes admitidos em UTIP Espírito Santo foi 14,13\%, resultado semelhante ao de Costa et al. (2010), onde a prevalência de mortalidade foi de 15\% em uma UTIP na cidade de São Paulo.

Ao avaliar se os desfechos, tempo de internamento e ter ido a óbito no internamento, estavam associados ao diagnóstico nutricional dos pacientes, não foi encontrada associação estatisticamente significante. O estudo de Batista et al. (2021) não observaram relação significativa de tempo de internação maior ou menor que 7 dias entre pacientes com ou sem desnutrição. Assim como o estudo de Vermilyea et al. (2013) onde não houve diferença significativa do estado nutricional com o tempo de internação na UTIP. Esses estudos corroboram com os resultados encontrados neste trabalho.

Diferentemente deste estudo, Bechard et al. (2016) encontrou que o quadro de desnutrição estava associado significativamente a maiores chances de infecções e mortalidade em 60 dias, assim como Prince et al. (2014) que avaliaram, no momento da admissão em UTIP, o estado nutricional de 12.458 pacientes pediátricos graves, foi fator de risco independente para mortalidade durante a internação. Porém, diante do tempo e do número de pacientes deste estudo, tais resultados podem ser insuficientes para uma análise mais adequada da situação, necessitando estudos complementares.

Não houve, nesse estudo, uma associação estatisticamente significante entre o estado nutricional e ser prematuro (p $<0,664$ ) e idade na admissão ( $\mathrm{p}<0,352$ ). Minamisava et al. (2004) constataram que $73 \%$ das crianças recém-nascidas prematuras nasceram com o peso adequado para idade gestacional. Um estudo de coorte populacional demonstrou que recémnascidos pré-termo tardios, tem um crescimento mais acelerado, que os a termo nos dois primeiros anos de vida (Rugolo, 2011). De acordo com a idade e estado nutricional, o estudo de Simões et al (2010), observou-se que a faixa etária dos lactentes apresentou valor mais baixo de escore $\mathrm{z}$ dos índices IMC/ idade quando comparado aos demais grupos ( $\mathrm{p}=0,001)$, diferente dos resultados encontrados neste estudo.

A avaliação do estado antropométrico dos pacientes internados em uma UTIP é fundamental, pois possibilita um maior conhecimento sobre os riscos nutricionais encontrados nesse setor e, os possíveis desfechos existentes nesses pacientes. As pesquisas de avaliação antropométrica no público específico de UTIP ainda são pouco difundidas, principalmente no Brasil, 
sendo necessário mais estudos que visem conhecer, identificar e explorar a realidade existente, possibilitando, assim, projetar futuras ações.

A ausência de associações encontradas nesse estudo, podem ser justificadas pela amostra relativamente baixa de pacientes, devido às limitações para realizar adequadamente a avaliação antropométrica em pacientes criticamente doentes internados em uma UTI pediátrica.

\section{Considerações Finais}

A avaliação do estado antropométrico dos pacientes críticos internados na UTI Pediátrica no presente estudo demonstrou elevado percentual de crianças desnutridas e não houve associação estatisticamente significante entre o estado antropométrico, idade, tempo de internamento e os desfechos tempo de internamento e óbito.

Sugere-se a elaboração de novos estudos neste público, dadas as particularidades dessa faixa etária, especialmente, no que tange ao cuidado em terapia intensiva. Assim recomenda-se para trabalhos futuros a avaliação constante do estado antropométrico em crianças internadas em estado crítico, pois essa verificação permite uma maior assertividade no diagnóstico e terapia nutricional, individualizando o tratamento e obtendo-se uma evolução desejada.

\section{Referências}

Batista, I. G. S., Gomes, A. C. V., Olinto, E. O. S. et al. (2021). Estado nutricional de pacientes críticos pediátricos: associação com o quadro clínico e desfecho terapêutico. Brazilian Journal of Health Review, Curitiba. 4(1), 1669-86.

Bejon, P.; Mohammed, S., Mwangi, I., Atkinson, S. H., Osier, F., Peshu, N., Newton, C. R.; Maitland, K. \& Berkley, J. A. (2008). Fraction of all hospital admissions and deaths attributable to malnutrition among children in rural Kenya. Am J Clin Nutr. 88, $1626-31$.

Bechard, L. J., Duggan, C, Touger-Decker, R, Parrot, J. S., Rothpletz-Puglia, P., Byham-Gray, L. et al. (2016). Nutritional status based on body mass index is associated with morbidity and mortality in mechanically ventilated critically ill children in the PICU. Crit Care Med. 44, 1530-7

Brooks, J. et al. (2011). Low weight, morbidity, and mortality in children with cerebral palsy: new clinical growth charts. Pediatrics. 128(2).

Cameron, J. W., Rosenthal, A. \& Olson, A. D. (1995). Malnutrition in hospitalized children with congenital heart disease. Arch Pediatr Adolesc Med. 149, 1098-102.

Costa, G. A., Delgado, A. F., Ferraro, A. \& Okay, T. S. (2010). Application of the pediatric risk of mortality (PRISM) score and determination of mortality risk factors in a tertiary pediatric intensive care unit. Clinics. 65(11), 1087-92.

Ferreira, H. S. (2000). Avaliação Nutricional de crianças pelo método antropométrico. In: Ferreira HS. Desnutrição, magnitude, significado social e possibilidade de prevenção. Maceió: Edufal;.2, 33-89.

Hecht, C., Weber, M., Grote, V., Daskalou, E, Dell'era, L., Flynn, D. et al. (2014). Disease associated malnutrition correlates with length of hospital stay in children. Clin Nutr. 34(1), 53-59.

Hulst, J., Joosten, K., Zimmermann, L., Hop, W., Van Buuren, S., Büller, H. et al. (2004). Malnutrition in critically ill children: from admission to 6 months after discharge. Clin Nutr. 23, 223-32.

Ista, E. \& Joosten, K. (2005). Nutritional assessment and enteral support of critically ill children. Crit Care Nurs Clin North Am. 17, $385-93$.

Jacquot, A., Valla, F. V., Mura, T., Tume, L. N., Bertet, H., Ford-Chessel, C., Milesi, C. et al. (2019). "Nutri-reaped study: nutritional assessment of French critically ill children and nutrition practice survey in French-speaking pediatric intensive care units." Annals of intensive care. 9(15), 2-11.

Jiménez, G. R. \& Santana, P. S. (2011) Estado nutricional de los niños atendidos en el Hospital Pediátrico “Juan Manuel Márquez", De La Habana (Cuba). Rev Cubana Aliment Nutr. 21(2), 236-47.

Lim, S. L., Ong, K. C. B., Chan, Y. H., Loke, W. C., Ferguson, M. \& Daniels, L. (2012). Malnutrition and its impact on cost of hospitalization, length of stay, readmission and 3-year mortality. Clin Nutr. 31(3), 345-50.

Mesquita, M., Iramain, R., Chavez, A., Avalos, S. \& Duarte, A. (2008). Estado nutricional em la unidad de Cuidados Intensivos Pediátricos: influye sobre la morbimortalidad? Pediatría (Asunción). 35, 88-94.

Minamisawa, R. et al. (2004). Fatores associados ao baixo peso ao nascer no Estado de Goiás. Revista Eletrônica de Enfermagem. 6, 336-49.

Netto, L. A., Muniz, V. M., Zandonade, E., Maciel, E. L. N., Bortolozzo, R. N., Costa, N. F. et al. (2014). Performance of the Pediatric Index of Mortality 2 in a pediatric intensive care unit. Revista Brasileira de Terapia Intensiva. 26(1), 44-50. 
Research, Society and Development, v. 10, n. 13, e545101320959, 2021

(CC BY 4.0) | ISSN 2525-3409 | DOI: http://dx.doi.org/10.33448/rsd-v10i13.20959

Oliveira, C. A. S., Pinto, F. C. C., Vasconcelos, T. B. \& Bastos, V. P. D. (2017). Análise de indicadores assistenciais em uma Unidade de Terapia Intensiva Pediátrica na cidade de Fortaleza/CE. Cad. Saúde Colet., Rio de Janeiro. 25 (1), 99-105.

Pawellek, I., Dokoupil, K. \& Kaletzo, B. (2008). Prevalence of malnutrition in paediatric hospital patients. Clin Nutr. 27(1), $72-76$.

Pereira A. S. et al. (2018). Metodologia da pesquisa científica. UFSM.

Prince, N. J., Brown, K. L., Mebrahtu, T. F., Parslow, R. C. \& Peters, M. J. (2014). Weight-for-age distribution and case-mix adjusted outcomes of 14,307 paediatric intensive care admissions. Intensive Care Medicine. 40(8), 1132-39.

Ribeiro, V. A. A., Thaisy, C. H. S. \& Fatal, L. B. S. (2017). Pacientes pediátricos hospitalizados: evolução do estado nutricional e fatores associados. BRASPEN J 2018. 33(1), 32-8.

Rocha, G. A., Rocha, E. J. M. \& Martins, C. V. (2006). Hospitalização: efeito sobre o estado nutricional em crianças. J. Pediatr. (Rio J.), Porto Alegre. 82(1), 70-74.

Rugolo, L. M. S. S. (2011). Manejo do recém-nascido pré-termo tardio: peculiaridades e cuidados especiais. Sociedade Brasileira de Pediatria. 1-13.

Sarni, R. O. S., Carvalho, M. F. C. C., Monte, C. M. G., Albuquerque, Z. P. \& Souza, F. I. S. (2009). Anthropometric evaluation, risk factors for malnutrition, and nutritional therapy for children in teaching hospitals in Brazil. J Pediatr. (Rio J). 85(3), 223-8.

Sigulem, D. M., Devincenzi, M. U. \& Lessa, A.C. (2000) Diagnóstico do estado nutricional da criança e do adolescente. J Pediatr. (Rio J). 76, $275-84$.

Silva, A. C.; Calmon, L. S.; Leite, L. O. \& Fatal, L. B. S. (2021). Adequação Calórico-Proteica e Nutrição Enteral Precoce em uma Unidade de Terapia Intensiva Pediátrica: um estudo observacional / caloric-protein adequacy and early enteral nutrition in an intensive care unit pediatric. Brazilian Journal Of Health Review, [S.L.], South Florida Publishing LLC. 4(3), 13124-37.

Silva, E. P. \& Tiengo, A. (2014). Perfil Nutricional de Crianças Hospitalizadas e sua Relação com o Período de Internação em um Hospital de Ensino no Sul de Minas Gerais. Revista Ciências em Saúde. 4(4), 3.

Silveira, C. R. M., Mello, E. D. \& Carvalho, P. R. A. (2008). Evolution of nutritional status of pediatric in patients of a tertiary care general hospital in Brazil. Nutr Hosp. 23(6), 599-606.

Simões, A. P. B. et al. (2010). Estado nutricional de crianças e adolescentes hospitalizados em enfermaria de cirurgia pediátrica. Rev Paul Pediatr. 28(1):41-7.

Souza, M. F., Leite, H. P. \& Koch, N. P. C. (2012). Malnutrition as an independent predictor of clinical outcome in critically ill children. Nutrition. 28(3):26770 .

Vermilyea, S., Slicker, J., El-Chamas, K., Sultan, M., Dasqupta, M., Hoffmann, R. et al. (2013). Subjective global assessment in criticallychildren. J Parenter Enter Nutr. (37):659- 666.

World Health Organization, Food and Agriculture Organization of the United Nations. (2018). Driving commitment for nutrition within the UN Decade of Action on Nutrition: policy brief. Geneva: World Health Organization; (WHO/NMH/NHD/17.11).

WHO Multicentre Growth Reference Study Group - WHO Child Growth standards based on length/height, weight and age. (2006). In: De Onis, M; Garza, C; Onyango, AW and Martorell, R, Guest Editors - WHO Child Growth Standards, Acta Paediatrica. 95 (450), 76-85.

World Health Organization. Growth reference data for 5-19 years, WHO reference (2007). < http://www.who.int/growthref/en/>. 